\title{
The use of periodic operation to improve the performance of continuous stirred tank reactors
}

\author{
A. RENKEN \\ Institut für Technische Chemie der Technischen Universität Hannover, D-3000 Hannover, Callinstraße 46, \\ Germany
}

(First received 4 October 1971; accepted 2 February 1972)

\begin{abstract}
The influence of enforced periodic concentration variations on a continuous stirred tank reactor was studied theoretically. The unsteady-state-processing becomes advantageous under certain conditions. This can be shown for an irreversible consecutive-competing reaction. The yield and selectivity of the intermediate product can exceed the values obtainable at steady-state-processing in the mentioned reactor.
\end{abstract}

\section{INTRODUCTION}

THE INFLUENCE of unsteady-state operation on technical processes has been under consideration and the object of experiments for some time. It has been shown that for separation processes such as extraction, distillation and adsorption an improvement in performance not only with respect to the quantity but also the quality of the output occurs with unsteady-state operation $[1,2]$.

There are also some published studies on the influence of periodic operation on homogeneous chemical reactions [3-12]. The parameters which can be periodically varied in continuous reactors are temperature, mean residence time, reactor volume, and feed-concentration of the reactants.

In general an influence of the varied parameters on the degree ' $y$ ' onversion can be expected in cases in which he reaction rate is a nonlinear function of this parameter.

In this paper only the effect of enforced periodic concentration variations in a continuous stirred tank reactor (CSTR) will be dealt with. In particular the effect of concentration variations in the feed of an isothermal CSTR is considered.

\section{SIMPLE SECOND ORDER REACTIONS}

In a second order reaction, the nonlinear dependence of the reaction rate on th ? concentration of reactants gives a different average reaction rate under oscillating conditions from that produced by equivalent but steady-state conditions. For example in the CSTR a higher timeaverage degree of conversion is achieved when the input concentration varies between say two and zero, than with steady-state operation and an input concentration of unity and otherwise identical conditions of temperature, volume and flow rate.

The influence of periodic concentration variations in the feed can be considered as follows. The reactant concentration varies as a square wave function. The input concentration has a value of two units during the first half period. In the second half the concentration is zero. Equation (1) gives the material balance for the CSTR.

$$
\begin{aligned}
\frac{\mathrm{d} c_{A}^{*}}{\mathrm{~d} \theta} & =c_{A 0}^{*}-c_{A}^{*}-k_{1} \cdot \tau \cdot \bar{c}_{A 0} \cdot c_{A}^{* 2} \\
\frac{\mathrm{d} c_{P}^{*}}{\mathrm{~d} \theta} & =-c_{P}^{*}+k_{1} \cdot \tau \cdot \bar{c}_{A 0} \cdot c_{A}^{* 2} \\
c_{A}^{*} & =c_{A} / \bar{c}_{A 0}, c_{P}^{*}=c_{P} / \bar{c}_{A 0}, \theta=t / \tau .
\end{aligned}
$$

Figure 1 shows the considered input function. The equations are dimensionless, since the concentrations of components $A$ and $P$ are referred to the average input concentration over a period $c_{A v}$. The time $\theta$ is referred to the mean residence time $\tau$ in the reactor.

At periodic operation the period $\theta^{\prime}$ is also referred to the mean residence time. This has the 


\section{A. RENKEN}

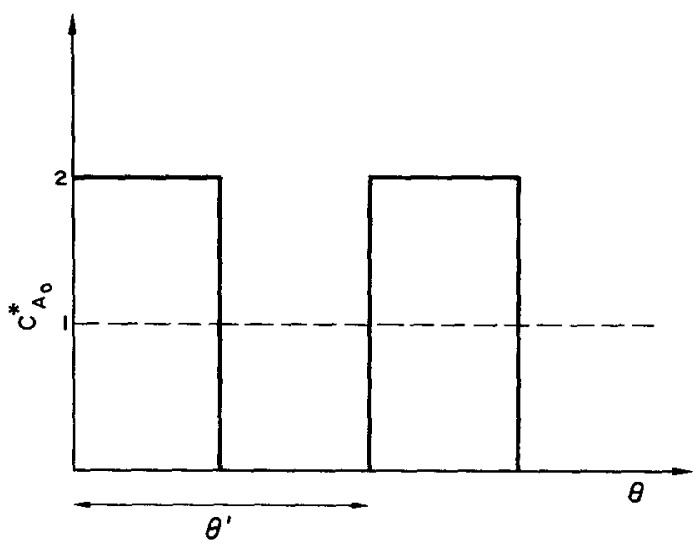

Fig. 1. Feed-concentration as a function of dimensionless time.

advantage that reference can be made with respect to the period or frequency independently of the value of the mean residence time, which varies with the reaction rate constant and the degree of conversion required.

In order to evaluate the differences between steady and non-steady state, i.e. periodic operation comparable conditions must be used. Periodic operation will then only be advantageous when the conversion of reactants to the desired product per time and volume unit can be increased. In other words for a given reactor vol$\mathrm{u}$ : $\leadsto$ e the amount produced in moles or $\mathrm{kg}$ in unit time must be the criterion for comparison. To achieve this the following conditions must be observed:

1. The amount (mass) of reactants which reaches the reactor in unit time, i.e. the molar rate of flow of component $A$ is constant.

2. The variable parameter, in this particular case the feed-concentration may not exceed the value for steady state operation.

The feed-concentration at periodic operation is therefore always smaller or the same as the concentration in steady-state operation and therefore the volumetric flow rate at periodic operation is higher than under steady-state conditions.

The concentration variations can be produced by the addition of a symmetrical input function and concentration variations between maximum values of two and zero, the mean residence time $\tau_{p}$ is only half of that in steady state operation, i.e. the flow rate of the feed is twice as large. When considering steady-state operation the residence time is adjusted for optimum results.

If one considers the extreme cases of very high and very low frequency it is clear that the time average degree of conversion must depend on the frequency of the concentration variations.

At high rates of change in concentration the reactor is no longer capable of following the imposed variations and the result is effectively that obtained from a constant input of unity. If the frequency falls to zero $\left(\theta^{\prime} \rightarrow \infty\right)$, a conversion corresponding to a constant input of concentration two is obtained. These results can be calculated as for the steady-state by using Eq. (2).

$$
\begin{aligned}
& c_{A 0}^{*}-c_{A}^{*}-k_{1} \bar{c}_{A 0} c_{A}^{* 2}=0 \\
& \theta \rightarrow 0: c_{A 0}^{*}=1, \tau=\tau_{p} \\
& \theta \rightarrow \infty: c_{A 0}^{*}=2, \tau=\tau_{p}
\end{aligned}
$$

optimal steady-state processing: $c_{A 0}^{*}=2, \tau=\tau_{0 D}$.

Figure 2 shows the results for various values of the period $\theta^{\prime}$. It can be seen that for increasing length of period, the average degree of conversion increases and tends to the limiting value mentioned above. Periodic operation, however

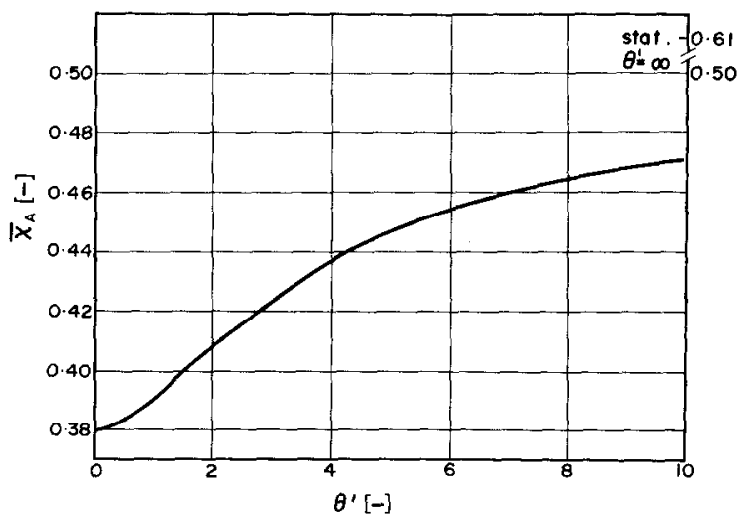

Fig. 2. Average degree of conversion $\bar{X}_{A}$ as a function of the length of period $\theta^{\prime}$ for a 2 nd order reaction; $k_{1} \cdot \tau_{0 p} c_{A 0}=4$ (stationary operation), $k_{1}, \tau_{p} \cdot \bar{c}_{A 0}=1$ (periodic operation). 
does not lead to improved results in this particular case. Under comparable conditions the degree of conversion is 22 per cent greater than the best available at periodic operation. The reason is the smaller mean residence time in the latter case.

\section{COMPLEX REACTIONS}

If one turns to more complex reaction-systems however, unsteady-state processing becomes advantageous. An example are consecutivecompeting reactions. The general form of such a reaction is given by the Eqs. (3):

$$
\begin{aligned}
& A+B \stackrel{k_{1}}{\longrightarrow} P \\
& P+B \stackrel{k_{2}}{\longrightarrow} R \\
& R+B \stackrel{k_{3}}{\longrightarrow} S \text { etc. }
\end{aligned}
$$

Examples of such reactions are the halogenation or nitration of hydrocarbons or the addition of alkene oxides, e.g. ethylene oxide, to compounds of the proton donor class, e.g. amines, alcohols or water.

In the following treatment it is assumed that the reaction stops after the second step, i.e. there is no reaction between $R$ and component $B$. Furthermore irreversible second order reactions are assumed. The desired product is the component $P$.

At steady-state process the yield of the intermediate product, i.e. the ratio of product concentration to input concentration, as a function of the degree of conversion of component $A$ goes through a maximum. The height of the maximum depends on the ratio of the rate constants $k_{1} / k_{2}=$ $x$. With increasing value of $x$ the maximum increases. The position of the maximum is also a function of this ratio. At larger ratios of $x$ the maximum occurs at higher degrees of conversion.

These maxima attainable at steady-state processing, must now be compared with the results at periodic operation.

A second important parameter, the selectivity must also be considered together with the yield. The relationship between yield, selectivity, degree of conversion and the mean residence time in a CSTR are given by the Eqs. (4)-(6).

$$
\begin{aligned}
X_{A} & =\left(c_{A_{0}}-c_{A}\right) / c_{A_{0}} \\
\sigma_{p} & =c_{p} /\left(c_{A_{0}}-c_{A}\right) \\
\eta_{p} & =c_{p} / c_{A_{0}}=\sigma_{p} . X_{A} .
\end{aligned}
$$

The optimum residence time which is required for the comparison of the two processes is given by Eq. (7).

$$
\tau_{0 p}=\frac{c_{A_{0}}-c_{A_{m}}}{k_{1} \cdot c_{A_{m}} c_{B_{m}}}
$$

The concentrations $c_{A_{m}}$ and $c_{B_{m}}$ indicate the values at the maximum yield in the CSTR.

The selectivity is also a function of the degree of conversion. In contrast to the yield, the selectivity does not pass a maximum for an intermediate product but falls from unity at small conversions to zero at complete conversion. When comparing periodic and steady-state operation therefore, the selectivity is taken at maximum yield. In the case of periodic operation always time-average values are considered.

The following differential Eqs. (8a)-(8d) were solved for yield, selectivity and conversion as functions of frequency in an analogue computer and numerical by the Runge-Kutta method [13] in a digital computer.

$$
\begin{aligned}
& \frac{\mathrm{d} c_{A}^{*}}{\mathrm{~d} \theta}=c_{A 0}^{*}-c_{A}^{*}-k_{1} \cdot \tau \cdot \bar{c}_{A 0} \cdot c_{A}^{*} \cdot c_{B}^{*} \\
& \frac{\mathrm{d} c_{B}^{*}}{\mathrm{~d} \theta}=c_{B 0}^{*}-c_{B}^{*}-k_{1} \cdot \tau \cdot \bar{c}_{A 0} \cdot c_{A}^{*} \cdot c_{B}^{*} \\
& -k_{2} \cdot \tau \cdot \bar{c}_{A 0} \cdot c_{B}^{*} \cdot c_{P}^{*} \\
& \frac{\mathrm{d} c_{P}^{*}}{\mathrm{~d} \theta}=-c_{P}^{*}+k_{1} \cdot \tau \cdot \bar{c}_{A 0} \cdot c_{A}^{*} \cdot c_{B}^{*} \\
& -k_{2} \cdot \tau \cdot \bar{c}_{A 0} \cdot c_{B}^{*} \cdot c_{P}^{*} \\
& \frac{\mathrm{d} c_{R}^{*}}{\mathrm{~d} \theta}=-c_{R}^{*}+k_{2} \cdot \tau \cdot \bar{c}_{A 0} \cdot c_{B}^{*} \cdot c_{P}^{*} .
\end{aligned}
$$

The variation of concentration of the reactants in a CSTR is shown in Fig. 3. Conditions have been chosen so that the reaction rate constants $k_{1}$ and $k_{2}$ are equal. The feed-concentration of 


\section{A. RENKEN}

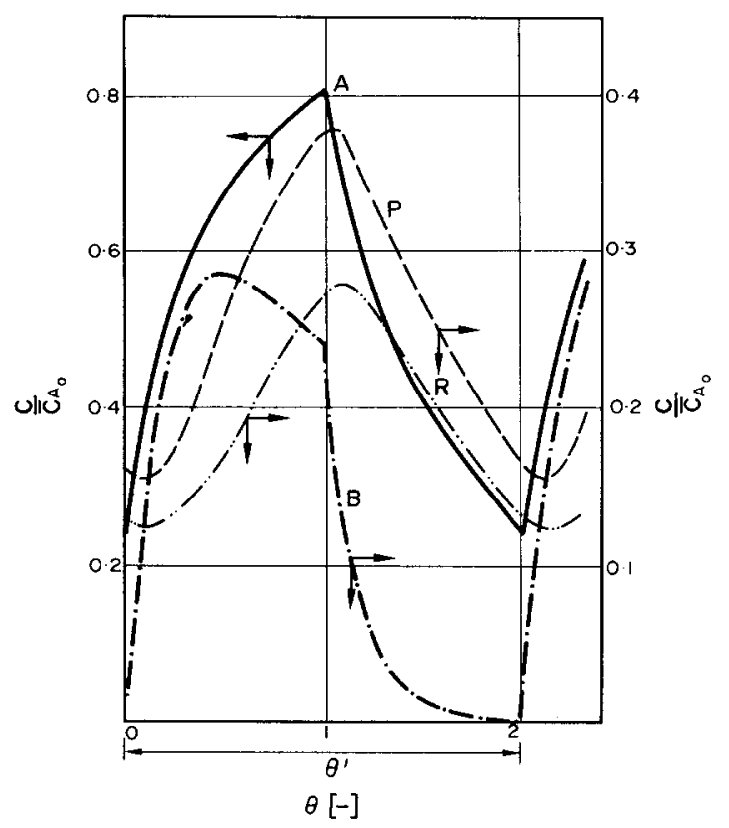

Fig. 3. Calculated dimensionless concentration of the reactants as a function of dimensionless time. $k_{1} / k_{2}=1$,

$$
\bar{c}_{B \mathrm{o}}=0 \cdot 8 \bar{c}_{A \mathrm{o}}, \theta^{\prime}=2 \text {. }
$$

reactant $B$ is 20 percent less than reactant $A$. The period of the square-ware function is $\theta^{\prime}=2$. It can be seen that the concentration of component $B$ very soon goes through a maximum. The reason is the increase in the rate of reaction to the next product $R$ caused by the growing concentration of $P$. A preferential formation of the intermediate product is the result of the displacement of this maximum. The advantage of this procedure can also be seen from the diagram (Fig. 3). Under optimal conditions at steadystate processing, a maximum yield of $\eta_{0}=0.25$ with a selectivity of $\sigma_{p}=0.5$ can be achieved, i.e. the concentration of both products is the same. In the case considered here the concentration of the undesired product is always less than the component $P$ through the wholc period. The degree of conversion is of course smaller than at steady-state processing but this is compensated and exceeded under certain conditions by the improved selectivity. So the yield of the intermediate can reach or exceed the values for steady-state processing.

At very high and very low frequencies the reactor behaves as a CSTR in the steady state, as in the previous simple case. Yield, selectivities and conversions are obtained as for at steadystate processing with average input conditions.

In Fig. 4 the dependence of yield and selectivity are shown as a function of the input period. The rate constants and feed-concentrations are equal. The maximum achievable yield in a stationary CSTR under these conditions is $\eta_{p}=0.25$ at a selectivity of $\sigma_{P}=0.5$.

For the limiting conditions when the period approaches zero $\bar{\eta}_{p}$ is 0.2267 and $\bar{\sigma}_{p}$ is 0.653 and when the period tends to $\infty, \bar{\eta}_{p}$ is 0.2454 and $\bar{\sigma}_{p}$ is

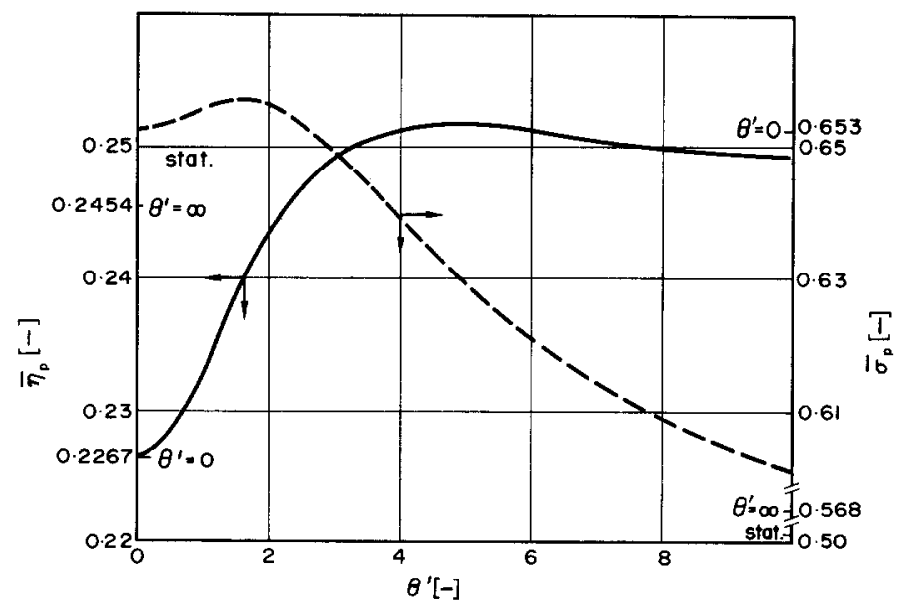

Fig. 4. Average yield and selectivity for the intermediate product as a function of the length of period. $k_{1} / k_{2}=$ $1, \bar{c}_{B 0}=\bar{c}_{A 0}, k_{1} \cdot \tau_{o p} \cdot c_{A 0}=4$ (stationary operation), $k_{1}, \tau_{p}, \bar{c}_{A 0}=1$ (periodic operation). 
0.568. Between these two limiting values the curves go through weak maxima which for the yield is about 0.5 per cent above that obtainable in the stationary CSTR. But more important is the simultaneous increase in selectivity. At a yield of $\bar{\eta}_{p}=0.25$ the selectivity is $\bar{\sigma}_{\nu}=0.648$ which is nearly 30 per cent higher than that at steady-state processing. Thus less of the non required product is formed and more unreacted starting material can be returned to the reaction.

The selectivity maximum occurs at a much smaller period at $\theta^{\prime}=1.8$ for the given conditions.

A different result is obtained when different conditions are used. For example, if the concentration ratio between $A$ and $B$ is changed, the result shown in Fig. 5 is produced. The ratio of the reaction rate constants is again unity in this example but the feed-concentration of component $B$ is 20 per cent smaller than the concentration of $A$. The calculations show a noticeable shift in the maxima for selectivity and yield to smaller periods. Furthermore the maximum in the yield curve is clearly larger, and with a value of $\bar{\eta}_{p}=$ 0.2656 some 6.3 per cent higher than the best value for steady-state processing. The selectivity on the other hand rises only to $\bar{\sigma}_{p}=0.574$, some 15 per cent better than in the stationary case under conditions of maximum yield.

Increasing rate constant ratio has a similar effect. The result of calculations for a ratio $x=16$ is shown in Fig. 6.

Figure 7 shows the optimum period as a function of the rate constant ratio and the feedconcentration ratio of unity. At a rate-constant ratio of $x=0.5$ the best period is $\theta^{\prime}=6$. This means that an almost stationary state obtains in the reactor before the input conditions are changed. The maximum yield of steady state processing cannot be achieved. An improvement in the selectivity is obtainable which cannot, however, be easily compared with the results from the stationary CSTR.

The change in yield with periodic processing in comparison to the steady-state processing is largely dependent on the kinetic parameters. In the extreme cases $k_{1} \ll k_{2}$ and $k_{1} \gg k_{2}$ the reaction cannot be influenced in the manner described and the results approach those obtainable in simple second order reactions. In other words in these cases periodic operation does not lead to improved yield or selectivity.

The dependence of the achievable yield on the rate constant ratio at optimum period is shown in Fig. 8.

By way of further comparison the yield achievable in a cascade with two CSTR designed for optimum performance [14] and an ideal plug flow reactor are also included. It can be seen that,

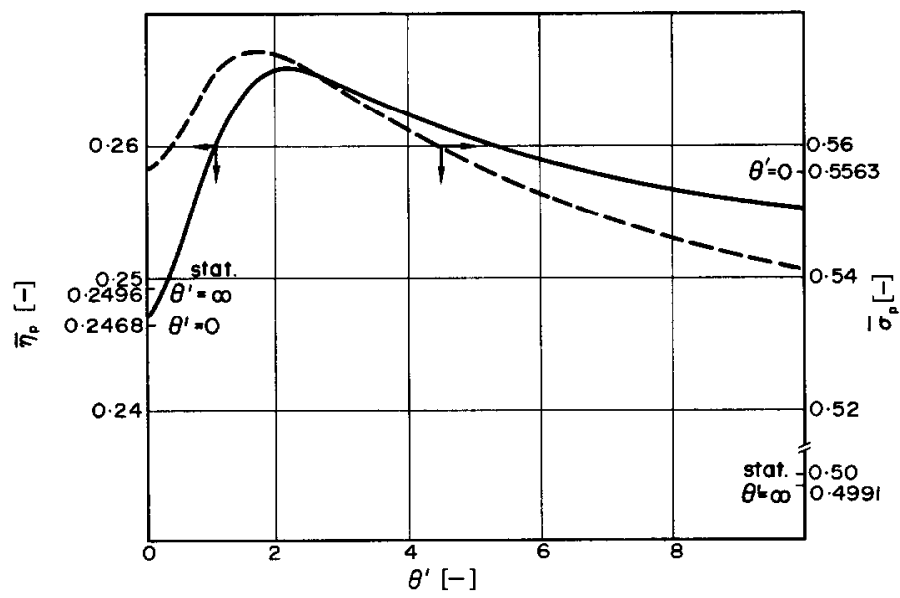

Fig. 5. Average yield and selectivity for the intermediate product as a function of the length of period, $k_{1} / k_{2}=$ $1, \bar{c}_{B 0}=0 \cdot 8 \bar{c}_{A 0}, k_{1}, \tau_{0 p}, c_{A 0}=10$ (stationary operation), $k_{1}, \tau_{p}, \bar{c}_{A 0}=2 \cdot 5$ (periodic operation). 


\section{A. RENKEN}

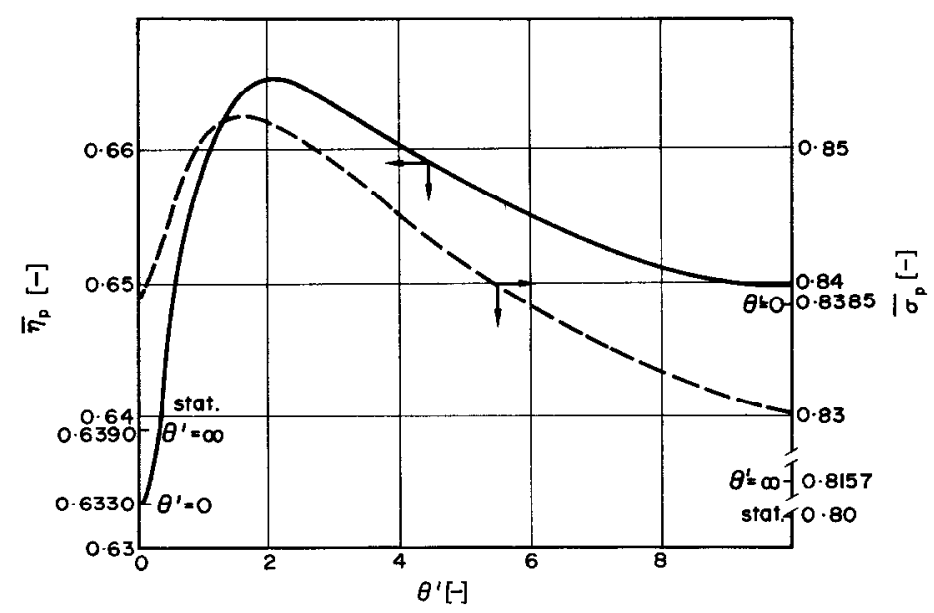

Fig. 6. Average yield and selectivity for the intermediate product as a function of the length of period. $k_{1} / k_{2}=16, \bar{c}_{B 0}=\bar{c}_{A 0}, k_{1} \cdot \tau_{0 p} \cdot c_{A 0}=100$ (stationary operation), $k_{1}, \tau_{p} \cdot \bar{c}_{A 0}=25$ (periodic operation).

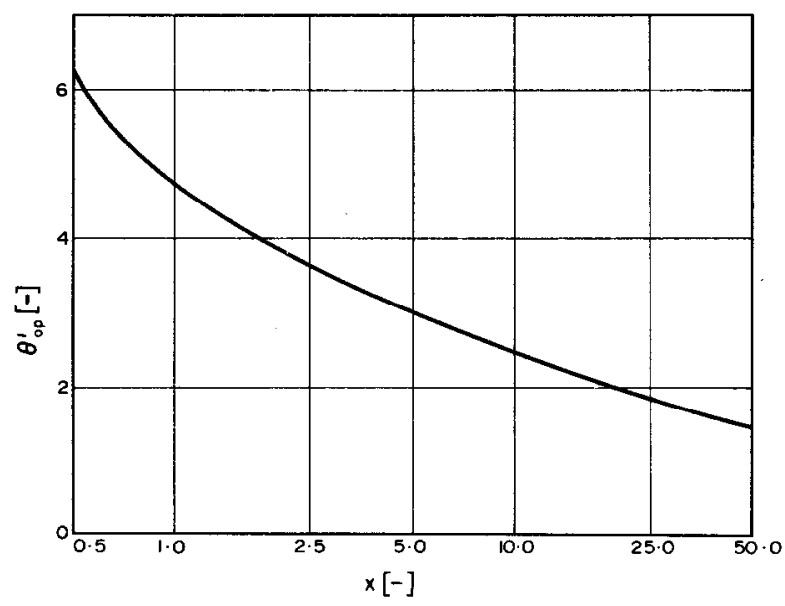

Fig. 7. Optimum length of period $\theta^{\prime}$ as a function of the rateconstant-ratio $x, \bar{c}_{A 0}=\bar{c}_{B 0}$.

depending on the rate constant ratio the yield of a CSTR can be improved by periodic processing methods. The yields are however noticeably smaller than in the cascade or plug flow reactor.

The selectivities obtainable at maximum yield as a function of the rate constant ratio for the four reactor types are shown in Fig. 9.

It is important to remark, that the selectivity in a periodic process is larger than the selectivity obtainable in an optimal cascade with two tanks up to $x=50$ and also in a plug flow reactor up to $x=6 \cdot 5$.

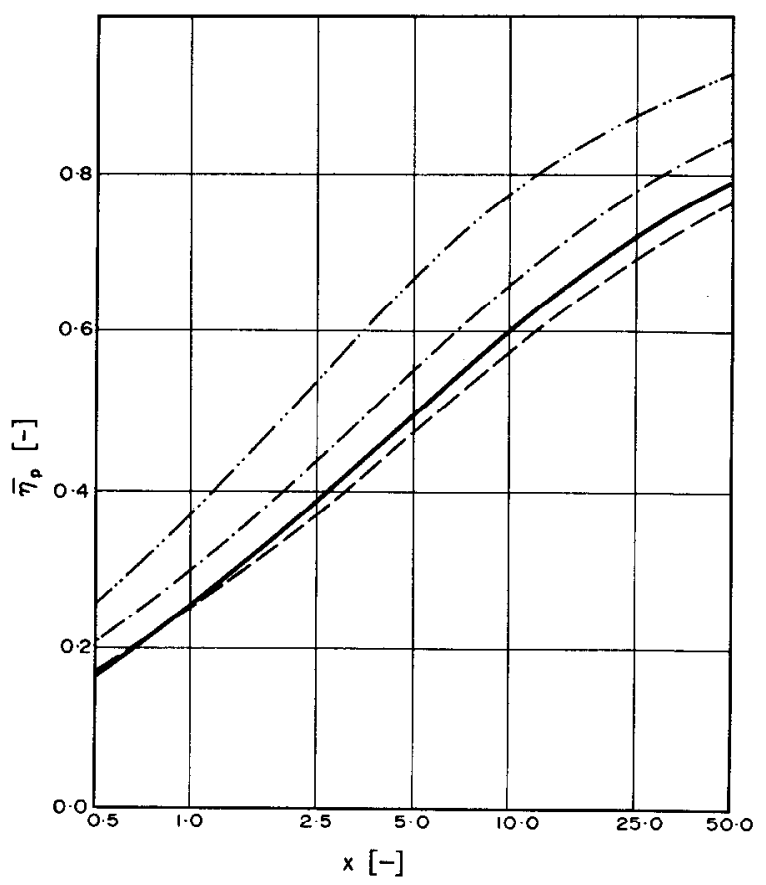

Fig. 8. Achievable yield at unsteady-state processing in a CSTR compared with steady-state-processing in a CSTR, an optimal cascade with two tanks, and a plug-flow reactor at steady-state as a function of the rate constant ratio. - periodic operation, --.-... CSTR, -...-. cascade with 2 CSTR, - -.-.- plug flow reactor.

\section{SUMMARY}

Compared with batch operation the yield of intermediate product obtainable in continuous 


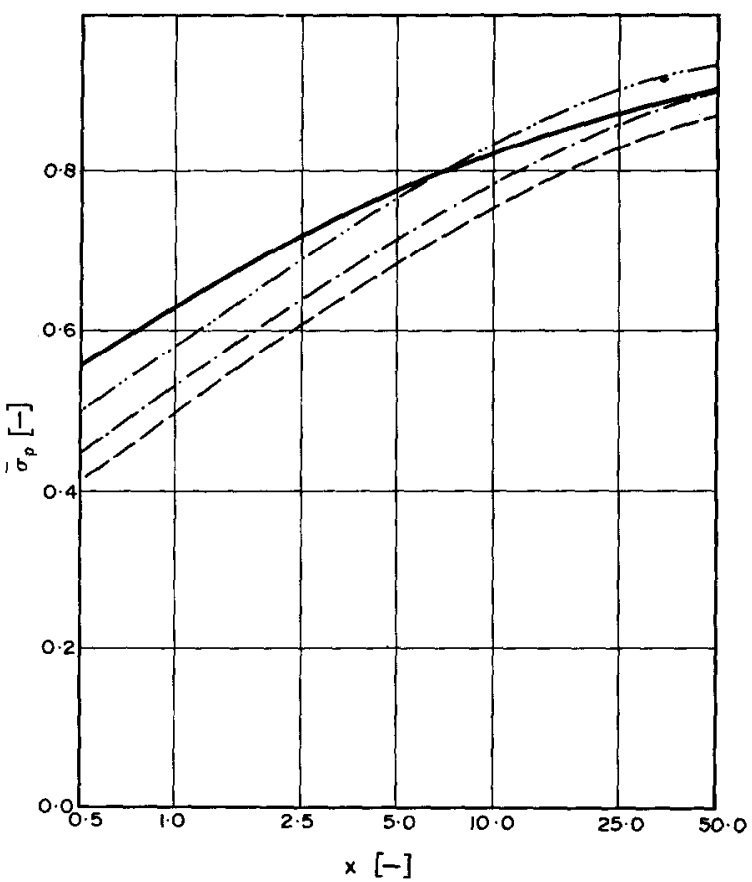

Fig. 9. Achievable selectivity at maximum yield at unsteadystate-processing in a CSTR and steady-state-processing in a CSTR, an optimal cascade with two CSTR, and a plug flow reactor as a function of the rate constant ratio. - _ periodic operation, -....... CSTR, -...-- cascade with 2 CSTR, -..-- plug flow reactor.

reactors as a CSTR or cascades with only a few tanks are considerably lower because of the smaller average concentrations which occur. But sometimes batch operation is difficult, particularly with fast exothermic reactions because of the high rate of reaction and heat release. In these cases CSTR are employed in spite of the low production rate of product. For very fast exothermic reactions, e.g. gas phase reactions, a cascade of CSTR is hardly possible. Instead of a
CSTR a loop-reactor might be used which corresponds to a CSTR at high recycle speeds. Periodic operation would certainly be of help in the maximisation of the production of intermediate products. The increased selectivity obtainable under such conditions is an additional advantage.

Acknowledgements - The author wishes to thank Mr. Dehnhardt and Dr. Viergutz for giving the computer-program of the Runge--Kutta Method, and Prof. Schügerl for his interest in this work.

This work was performed under the support of the Deutsche Forschungsgemeinschaft.

\section{NOTATION}

c concentration, $\mathrm{g}$ mole/vol

$c^{*}$ concentration referred to the feed-concentration, dimensionless

$k_{i}$ reaction-rate-constant of the $i$ th reaction, vol./(g mole . sec)

$t$ time, sec

$x \quad k_{1} / k_{2}$, reaction-rate-constant-ratio

$X$ conversion, dimensionless

Greek symbols

$$
\begin{aligned}
\eta & \text { yield } \\
\theta & t / \tau, \text { dimensionless time } \\
\theta^{\prime} & \text { length of period, dimensionless } \\
\sigma & \text { selectivity } \\
\tau & \text { mean residence time, sec }
\end{aligned}
$$

\section{Subscripts}

$$
\begin{aligned}
A, B & \text { reactants } \\
P, R, S & \text { products } \\
0 & \text { feed conditions } \\
0 p & \text { optimal conditions } \\
p & \text { conditions at periodic operation }
\end{aligned}
$$
overbar denotes average values

[1] SCHRODT V. N., Intn. Sci. Techn. 196762.

[2] SCHRODT V. N., Ind. Engng Chem. $5919676 ; 58$.

[3] DOUGLAS J. M. and RIPPIN B. W. T., Chem. Engng Sci. 211966265.

[4] DOUGLAS J. M. and RIPPIN B. W. T., Ind. Engng Chem. Proc. Des. Dev. 6196743.

[5] DOUGLAS J. M., RIPPIN B. W, T. and GAITONDE N. Y., Ind. Engng Chem. Fundls. 61967265.

[6] HORN F. J. M. and LIN R. C., Ind. Engng Chem. Proc. Des. Dev. 6196721.

[7] CHANG K.S. and BANKOFF S. G., Ind. Engng Chem. Fundls. 71968633.

[8] FJELD M. and KRISTIANSEN T., Int.J. Control. 101969 6; 601.

[9] BACCARO G. P. et al., A.I.Ch.E.Jl 161970249. 


\section{A. RENKEN}

[10] CODELL R. B. and ENGEL A. J., A.I.Ch.E. Jl. 171971220.

[11] LUND M. M. and SEAGRAVE R. C., A.I.Ch.E.Jl17 197130.

[12] LUND M. M. and SEAGRAVE R. C.,J. EC Fundls 101971494.

[13] BRÄUNING G., Gewöhnliche Differentialgleichungen. Frankfurt/Main-Zürich 1964.

[14] KERBER R. and GESTRICH W., Chem.-Ing. Techn. 401968129.

Résumé - Les auteurs étudient en théorie l'influence de variations périodiques forcées de la concentration sur un réacteur-agitateur à écoulement continu. Le procédé en état instable devient avantageux dans certaines conditions. Ceci est démontré dans le cas d'une réaction opposée irréversible et consécutive. Le rendement et la sélectivité du produit intermédiaire peuvent dépasser ceux obtenus du procédé en état stable dans le réacteur décrit.

Zusammenfassung-Die Beeinflussung von Ausbeute und Selektivität in einem kontinuierlichen Rührkesselreaktor durch aufgezwungene periodische Konzentrationsschwankungen wurde theoretisch untersucht. Unter bestimmten Bedingungen bringt die instationäre Prozeßführung Verbesserungen. Dies kann anhand einer irreversiblen konkurrierenden Folgereaktion gezeigt werden. Ausbeute und Selektivität des Zwischenproduktes können die bei stationärer Prozeßführung erreichbaren Werte deutlich überschreiten. 